\title{
La flûte nasale des indiens Nambikuara
}

\section{Desidèrio Aytai}

Traducteur : Isabelle Schulte-Tenckhoff

\section{(2) OpenEdition}

\section{Journals}

Édition électronique

URL : http://journals.openedition.org/ethnomusicologie/2336

ISSN : 2235-7688

\section{Éditeur}

ADEM - Ateliers d'ethnomusicologie

\section{Édition imprimée}

Date de publication : 1 janvier 1989

Pagination : 133-149

ISBN : 2-8257-0178-5

ISSN : $1662-372 X$

\section{Référence électronique}

Desidèrio Aytai, "La flûte nasale des indiens Nambikuara », Cahiers d'ethnomusicologie [En ligne], 2 | 1989, mis en ligne le 15 septembre 2011, consulté le 02 mai 2019. URL : http://

journals.openedition.org/ethnomusicologie/2336 


\title{
LA FLÛTE NASALE DES INDIENS NAMBIKUARA *
}

\author{
Desidério Aytai
}

L'objectif de cet article est de décrire la flûte nasale des Indiens Nambikuara (État du Mato Grosso, Brésil), instrument rare dans les deux sens du terme, car peu de groupes indiens le connaissent, et même les Nambikuara n'en jouent que rarement.

La fig. 1 donne une idée générale de cet instrument: deux calottes sphériques de taille égale sont découpées dans une calebasse, puis collées par les bords; l'une d'elles est ensuite percée de trois trous de diamètre plus ou moins égal. Les noms que nous avons donnés aux diverses parties de l'instrument pour en faciliter la description sont indiqués dans le schéma.

\section{Les matériaux}

D'une manière générale, la flûte nasale est fabriquée à partir d'une calebasse dont il existe de nombreuses variétés. Or, comme l'instrument ne peut être confectionné avec de petites calebasses et que les variétés plus grandes sont plutôt rares, les Indiens emploient souvent des disques ayant une surface irrégulière, si bien que la ligne que nous avons dénommée «équateur» en fig. 1, censée former un cercle parfait, est déformée pour adapter les deux éléments et que, vue de côté, elle ressemble à un 8 . L'épaisseur de la paroi de l'instrument varie entre 3,5 et $6 \mathrm{~mm}$. Les bords des deux disques sont biseautés pour faciliter la pénétration de l'adhésif (voir A en fig. 1). Les soixante-treize flûtes nasales de notre collection ont été collées avec de la cire, mais les informations divergent à ce sujet, faisant état également de résine ou de colophane. Précisons à ce propos que les données disponibles ont été recueillies sur une période de plus de cinquante ans et que les divers groupes Nambikuara sont susceptibles de recourir à différents types d'adhésifs. La résine est facile à obtenir, surtout à

* Traduit de l'allemand par Isabelle Schulte-Tenckhoff. 
partir de l'écorce de l'arbre jatobá (Hymenaea stygonocarpa, Hymenaea courbaril), mais en quantité insuffisante. La cire d'abeilles jatai (Melipona lineata Lap.) est plus abondante, car il s'agit d'un produit secondaire de la «chasse au

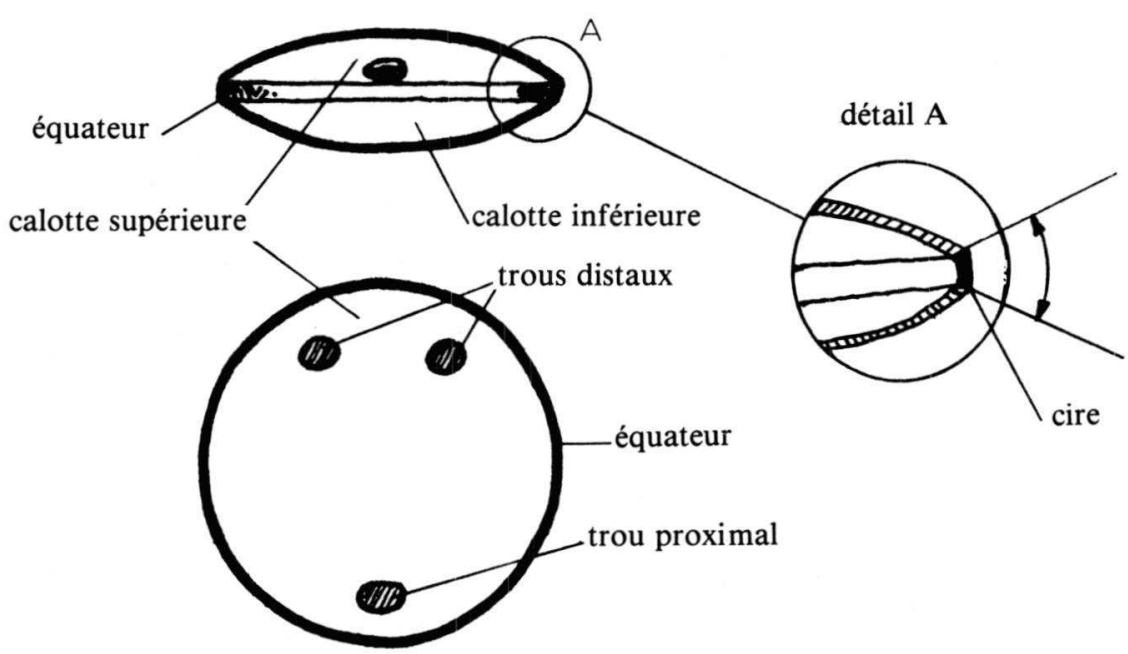

Fig. 1

miel», comme l'expriment les Indiens. Il convient de souligner cependant que l'abeille jatai est plus répandue dans les forêts humides subtropicales de l'État de Paraná que sur les hauts plateaux arides de l'État du Mato Grosso, ce qui explique notamment pourquoi les Xetá (Sheta) étaient si riches en cire, dont ils confectionnaient de nombreuses figures (cf. Kozák, Baxter, Williamson \& Carneiro 1979: 418, 424), alors que les Nambikuara étaient contraints d'économiser ce matériau qu'ils utilisaient aussi pour le collage des plumes sur les flèches.

Le miel est séparé de la cire de la manière la plus simple: les Nambikuara prennent un rayon de miel dans la bouche, le mâchent, avalent le miel et conservent la cire en boulettes, qui sont ensuite chauffées et réunies en boules plus grandes. Le collage s'effectue en chauffant simultanément la cire sur la pointe d'un bâtonnet et les disques découpés dans la calebasse (Aytai 1981: 107). C'est la fumée qui donne à la cire sa teinte brun foncé ou noir; le procédé de nettoyage de la cire utilisé par d'autres Indiens (Bororo, par exemple; voir Albisetti-Venturelli I, 1962: 808 «Muiávo bóri») n'a pas pu être identifié chez les Nambikuara. Quant aux sections de calebasse, leur couleur varie entre le doré et le brun foncé, voire même le noir. 


\section{Dimensions et décors}

Les principales dimensions des flûtes nasales de notre collection ont été réunies dans le tableau 1.

\section{Tableau 1: Dimensions des flûtes nasales}

\begin{tabular}{lcc} 
Dimensions en millimètres & $\max$ & $\min$. \\
\hline diamètre & 97 & 51 \\
épaisseur du disque & 39 & 16,7 \\
diamètre du trou proximal & 9 & 4,7 \\
diamètre des trous distaux identiques & 7 & 4,5
\end{tabular}

Les mensurations ci-dessus ont été faites sur des flûtes nasales venant des Nambikuara-Halotesu du village de Serra Azul. Les flûtes des Mamaindê (jusqu'en 1964) et des Wasusu-Galera (jusqu'en 1967) ont plus ou moins les mêmes dimensions, mais contrairement à celles des Halotesu, elles ne portent pas de pyrogravures. Une flûte nasale photographiée par Lévi-Strauss (1948: 370 , pl. 36 , photo en haut à droite) semble avoir un diamètre nettement plus grand que $97 \mathrm{~mm}$. Or, aucun des groupes que je connais ne confectionne aujourd'hui de flûtes aussi grandes.

Sur la plupart des instruments, le trou proximal est un peu plus grand que les trous distaux, bien qu'il existe aussi des flûtes dont les trois trous ont le même diamètre. La distance entre les trous et entre ceux-ci et le centre de la flûte peut varier.

Le plus souvent, soit dans $91 \%$ des cas, la section supérieure de la flûte est décorée de pyrogravures, contrairement à la section inférieure pour laquelle ce taux ne s'élève qu'à $14 \%$. Nous avons identifié douze types de dessins pour la partie supérieure, et trois pour la partie inférieure (voir fig. 2, où les premiers sont identifiés par des lettres majuscules, les seconds par des minuscules).

Les lignes radiales partant des trous, qui semblent reproduire des étoiles, se retrouvent sur $65 \%$ des flûtes décorées. Il s'agit probablement d'un dessin très ancien servant entre autres à dissimuler les imperfections des perces effectuées au moyen d'un outil chauffé. Sur le seul spécimen acquis par Boglár, les trois trous portent un dessin radial (1966: photo entre p. 144 et 145; Halmos 1979: 344), tandis que sur les photographies reproduites par Roquette-Pinto, l'instrument est dépourvu de décor (1938: 283, fig. 67).

Un autre type de dessin est constitué de points que l'on retrouve dans $41 \%$ des cas. Ils forment vraisemblablement une tradition distincte. Roquette-Pinto a publié huit types de décors propres aux «Indios da Serra Azul» (1938: 259-66), parmi lesquels cinq montrent des points, ce qui semble indiquer que les divers groupes indiens connaissent ce dessin depuis fort longtemps. Ainsi les peintures faciales et corporelles comprennent-elles souvent des points appliqués sous les lignes parallèles. A Serra Azul, nous avons vu plusieurs jeunes 

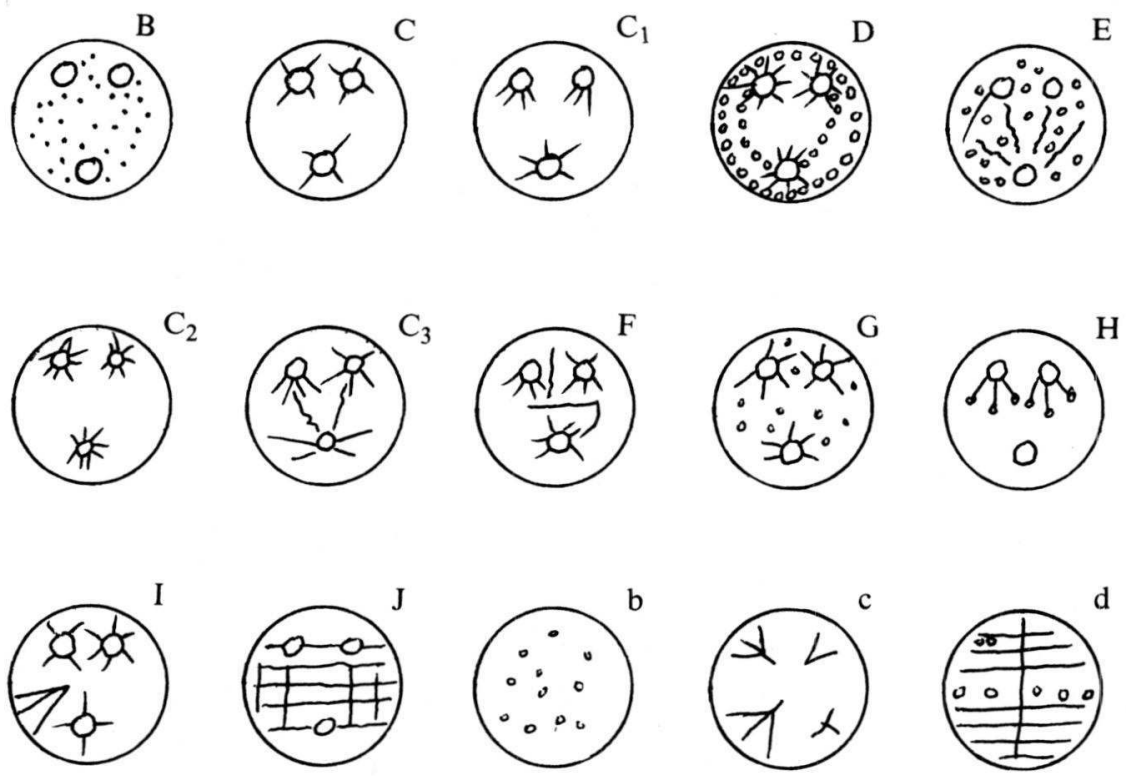

Fig. 2

femmes et jeunes filles qui s'étaient peint le visage et le ventre de cette manière (fig. 3); le dessin en question apparaît également sous une forme tridimensionnelle sur un vase en céramique de l' «Abrigo do sol», lieu de fouilles archéologiques où habite aussi un groupe Nambikuara (Puttkamer 1979: 60). Le type $d$ de la fig. 2 est une variante du même motif. Le triangle ou la fourche qui apparaît dans les types I et c, interprété comme un symbole sexuel féminin, se rencontre également à l'«Abrigo do sol» (Puttkamer 1979: 67, 77, 78). En revanche, les lignes courbes, difficiles à pyrograver, manquent sur tous les décors.

\section{Procédé de fabrication}

La fabrication de la flûte nasale est relativement simple et toujours effectuée par les femmes (Aspelin, communication personnelle 1971, et nos propres observations), parmi lesquelles il existe de véritables spécialistes en la matière. Tout d'abord, on découpe deux calottes sphériques plus ou moins égales dans une calebasse, de nos jours à l'aide d'un couteau, jadis au moyen d'un outil en silex ou d'un ciseau fait de la dent d'un cabiai (ou capivara, Hydrochoerus hydrochoeris L.) fixée sur un bâtonnet (Boglár 1966: photo entre p. 96 et 97). 

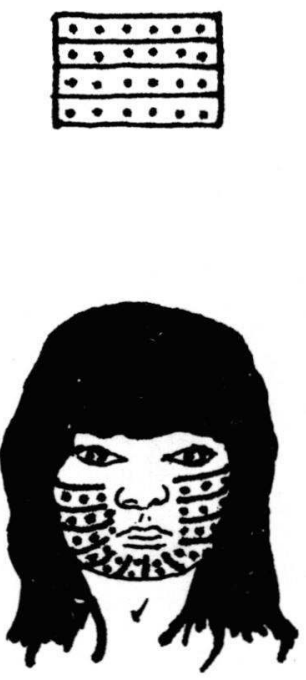
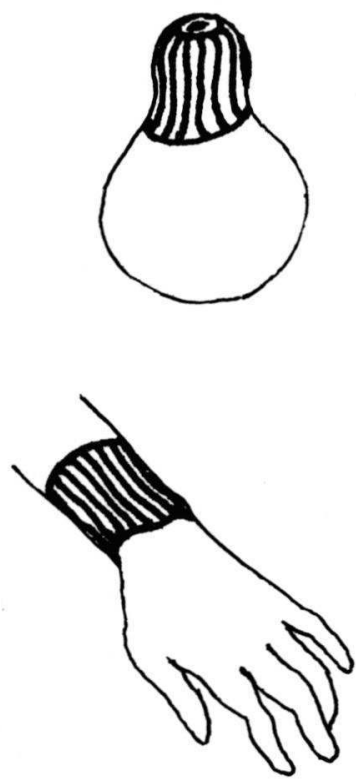

Fig. 3

Les deux outils sont très pratiques et plus faciles à manier que notre couteau - ce dont nous avons fait nous-même l'expérience (voir aussi LamingEmperaire, Menezes \& Andreatta 1978: 77, photo 3). Les sections découpées sont un peu plus grandes que nécessaire, car leurs bords doivent être cassés obliquement (fig. 1), travail moins ardu que la découpe de la surface externe résistante de la calebasse. Ensuite, les deux calottes sphériques sont opposées, la surface convexe à l'extérieur, et affilées longuement au moyen d'une pierre en grès jusqu'à ce qu'elles soient adaptées.

Parfois, la surface interne des deux disques est enduite d'un liquide noir que nous n'avons pas pu identifier, servant vraisemblablement à protéger l'intérieur, moins résistant, contre les insectes. Les deux sections sont ensuite accollées avec de la cire chaude. Les perces ne sont effectuées que sur l'instrument fini, comme on peut le remarquer encore sur la plupart des flûtes, car l'outil employé à cet effet endommage légèrement la face interne du disque inférieur, et de petits éclats qui n'ont pu être évacués par les trous séjournent ainsi à l'intérieur de la flûte.

De nos jours, les Indiens se procurent sans difficulté des clous ou du fil de fer qui, une fois chauffés à rouge, facilitent la perce des trous - travail jadis nettement plus ardu. Nous avons essayé de forer des trous à la manière ancienne, en utilisant pour cela des tisons de différentes espèces, mais sans 
succès. Quand on appuie le bois contre la surface de la calebasse, la flamme s'éteint; quand on essaie de la souffler, elle se répand. Les bois durs sont plus efficaces, mais le travail est fastidieux et exige de la patience, ce qui explique l'existence de spécialistes. Quelques-uns des instruments de notre collection n'ont pas été percés à l'aide du feu mais par des moyens mécaniques.

La forme du trou proximal a une grande influence sur le timbre, comme nous avons pu le constater aussi pour les instruments de musique d'autres Indiens (Xavante, Waurá) (Aytai 1967-68: 69). Il n'est pas cylindrique, comme il y paraît à première vue, et sa description géométrique par des équations est impossible. En outre, sa grandeur varie selon les flûtes. De même, la hauteur du son des flûtes nasales de notre collection varie considérablement de l'une à l'autre, tout comme leur ambitus.

\section{La technique de jeu}

La première description détaillée de la technique de jeu de la flûte nasale se trouve dans l'étude de Max Schmidt sur les Indiens Pareci (Schmidt 1912: 173 infra):

«Le petit instrument rond et plat fabriqué à partir d'une calebasse consiste en deux disques collés ensemble avec de la résine; il est muni, outre le trou dans lequel on souffle avec le nez, de deux trous de jeu. En jouant, le musicien bouche la narine droite avec le pouce droit, tandis qu'il actionne l'instrument avec le souffle de la narine gauche».

Roquette-Pinto (1938: 284) montre par une illustration comment on joue de la flûte nasale chez les "Indiens de la Serra do Norte», et sa description concorde avec celle de Max Schmidt. Si elle n'indique pas avec quel doigt on bouche la narine droite, l'auteur précise néanmoins que «les trous de la flûte sont obturés avec les deux majeurs». Lévi-Strauss fournit à peu près les même informations (1948: 368) qu'il complète par une photographie (pl. 26) corroborant le dessin de Roquette-Pinto, sur laquelle on voit clairement que la narine est tenue fermée avec le pouce droit. La description donnée par Oberg (1953: 101 infra) est identique à celle de Schmidt:

Boglár décrit l'instrument comme les auteurs susmentionnés, tout en ajoutant que les trous distaux sont fermés par les index (1966: 124), comme cela apparaît aussi sur les illustrations (1966: 144, 145). Halmos reprend la description de Schmidt en traduction anglaise, et il affirme en outre que la narine droite est bouchée avec le pouce gauche - contresens qui résulte probablement d'une faute typographique.

Comme le montrent ces citations, la seule divergence entre les auteurs consultés au sujet de la technique de jeu réside dans le fait que les uns mentionnent les deux index, les autres les deux majeurs. Il se peut que l'instrument n'ait pas été joué de la même manière parmi les différents groupes Nambikuara, ou que la technique de jeu se soit modifiée avec le temps. L'autre possibilité serait que, 
même au sein d'un seul groupe, la technique de jeu ait été soumise à de légères variations. Bien que toutes ces possibilités existent, nous croyons que la raison principale de la diversité des techniques de jeu doive être cherchée ailleurs. Quand nous avons commencé à apprendre à jouer de la flûte nasale, nous avons constaté que les instruments plus grands pouvaient être joués avec les majeurs, alors que les instruments plus petits exigeaient l'emploi des index pour éviter que les doigts ne touchent le nez et perturbent le jeu en général. Il s'ensuit que les photographies tout comme les dessins sont corrects. Sur la photo reproduite par Lévi-Strauss, l'instrument est grand et il est peut-être joué avec les majeurs, alors que celui reproduit par Boglár est petit et exigerait donc l'utilisation des index. Chez Roquette-Pinto, enfin, l'instrument est de taille moyenne, ses trous distaux pouvant ainsi être obturés avec les index ou les majeurs.

Dans la plupart des cas, le pouce couvre la narine en appuyant sur l'aile du nez; or en 1967, ma femme a observé à Serra Azul un flûtiste qui jouait de son instrument en insérant la pointe du pouce dans la narine.

A l'exception d'Oberg, aucun des auteurs cités ne précise que le musicien souffle, non pas dans mais par-dessus la flûte, si bien que son souffle ne fait qu'effleurer le trou dont l'arête le divise alors en deux flux: l'un frôle la surface, tandis que l'autre est dirigé à l'intérieur de l'instrument; là où s'effectue le partage se créent des vibrations que l'oreille perçoit comme des sons.

Quant aux doigtés, il n'existe que trois positions de jeu:

- position I: les deux trous sont fermés - ton le plus grave;

- position II: un trou est ouvert, l'autre fermé - ton moyen;

- position III: les deux trous sont ouverts - ton le plus aigu.

Une analyse statistique effectuée sur trente-neuf flûtes de notre collection, à sonorité belle et intense, a révélé que la plupart des flûtes émettent trois tons à des intervalles de 5 et de 3 . Le nombre des tons possibles est cependant beaucoup plus élevé pour plusieurs raisons. Non seulement un courant plus fort overblow en anglais - permet d'augmenter la hauteur. D'autre part, lorsqu'un ouvre très peu l'un des trous en position I, le ton est un peu plus aigu, et plus le trou est ouvert, plus le ton devient aigu jusqu'à ce que - avec le trou grand'ouvert - on en arrive à la position II - principe qui s'applique aussi aux positions II et III. De cette manière, il devient possible, du moins en théorie, de jouer tous les tons de l'instrument entre le plus grave et le plus aigu, avec des modulations infimes entre eux. Le flûtiste peut ainsi jouer portamento et glissando, principe qui, bien entendu, s'applique jusqu'à un certain point à n'importe quelle flûte, mais non pas nécessairement au même degré qu'à l'instrument des Nambikuara. Dans son ouvrage déjà cité, Halmos analyse les difficultés que les ethnmomusicologues n'ont cessé de rencontrer dans l'étude de cette musique. Grâce à quatre transcriptions fort détaillées de musique de flûte nasale, l'auteur a su identifier plus de tons que les trois tons principaux correspondant aux trois doigtés. Dans sa «Piece $\mathrm{N}^{\circ} 25 »$, par exemple, il n'indique pas moins de huit tons qui ont véritablement pu être reproduits en laboratoire. 


\section{Fonctions et significations}

La flûte nasale, telle que nous venons de la décrire, se retrouve parmi de nombreux peuples à travers le monde, mais elle ne nous paraît pas moins étrange. On se pose donc à juste titre la question de savoir pourquoi les Nambikuara recourent à cette technique, si l'on considère qu'ils possèdent des flûtes en bambou de diverses tailles et même une flûte de Pan, toutes jouées avec la bouche (pour la flûte de Pan, voir Lévi-Strauss 1948: pl. 6, photo à gauche dans la rangée inférieure). Dans sa contribution déjà citée, Oberg écrit:

«A côté des flûtes wáinrhu, les Nambicuara [sic] possèdent une flûte en bambou plus petite percée de quatre trous de jeu, kadinsu, la flûte nasale maitétansu et la flûte de Pan à cinq tubes appelée dúta» (1953: 101-102).

Comment expliquer le fait qu'une de ces flûtes soit soufflée par le nez? Ce problème a déjà été soulevé, il y a plus d'un demi-siècle, par Izikowitz dans son ouvrage magistral sur les instruments de musique des Amérindiens:

«Comment en sont-ils venus à l'idée de jouer d'une flûte par le nez? Je n'en sais rien. Selon Sachs [...] cette technique comporte une signification symbolique qui ne nous est toutefois pas confirmée par les Indiens euxmêmes») (1935: 328).

Quand nous avons appris à jouer de la flûte Nambikuara, nous avons souvent joué avec la bouche au lieu du nez, technique avec laquelle on peut produire les même sons. Or, nous avons rapidement été amené à constater que dans ce dernier cas, les poumons résistent moins longtemps sans respirer que dans le jeu nasal. L'idéal consistant à ne jamais respirer, et vu la difficulté qu'éprouvent les Indiens à prolonger leur jeu de la flûte nasale, force est de constater que la technique requérant le moins de souffle est la meilleure. La fréquence de la respiration dépend de nombreux facteurs: du musicien, de son instrument et de sa technique, de la mélodie, de l'acoustique du lieu... En ce qui concerne le musicien, sa capacité pulmonaire ou maximum aerobic power (Weiner \& Lourie 1961: 225) pourrait être chiffrée approximativement, mais de multiples facteurs entrent en ligne de compte ici, sans parler de la nonpertinence, en cette matière, de l'approche mathématique, si bien qu'on se voit contraint d'expérimenter au lieu de calculer. Sans prétendre que notre expérimentation ait produit des résultats exhaustifs, nous pouvons constater qu'en jouant avec la bouche, nous respirons plus souvent (30 à 45\%) qu'en jouant avec le nez. L'économie du souffle représente donc du moins une des raisons pour laquelle les Nambikuara possèdent une flûte nasale.

Nous pensons également que le souffle passant directement des poumons à la bouche provoque en cas de temps froid plus de condensation à l'intérieur de la flûte que le souffle déjà refroidi par les fosses nasales. Or, il fait souvent froid au pays des Nambikuara; selon nos journaux, nous avons relevé à Serra Azul une température de $7^{\circ} \mathrm{C}$ à l'aube, atteignant $32^{\circ} \mathrm{C}$ à l'ombre vers deux heures de l'après-midi. 
L'attribution d'une signification symbolique à la flûte nasale, comme le suggère Sachs, offre une autre voie pour comprendre pourquoi la flûte est jouée avec le nez. Comme on le sait (Aytai 1967-68), les Nambikuara possèdent aussi des flûtes en bambou jouées avec la bouche et utilisées lors des cérémonies religieuses pour interpréter des hymnes et des louanges à leur héros culturel, un jeune garçon qui a morcelé son corps pour créer ainsi les plantes utiles. D'un autre côté, comme nous l'étayerons plus loin, certains prétendent que les mauvais esprits de la forêt sont chassés au moyen de la flûte nasale; il semble dès lors logique que ces deux actes opposés soient accomplis au moyen de deux instruments de musique foncièrement différents.

Une autre question théorique à laquelle il nous faut répondre consiste à savoir pourquoi les Nambikuara possèdent une flûte nasale en forme de disque dont la fabrication exige un travail considérable, alors que de nombreux groupes du Mato Grosso utilisent la petite flûte globulaire insufflée avec la bouche, qui est plus facile à fabriquer. Ce dernier type de flûte est largement répandu parmi les Indiens du Brésil, et nous avons vu au Musée de Lagoa Santa une petite flûte globulaire en céramique percée de trois trous et en avons même joué, ce qui veut dire que ce type était déjà connu il y a plusieurs millénaires. D'un autre côté, le sol, les précipitations et le climat du pays des Nambikuara diffèrent notablement de ceux des autres régions brésiliennes; de plus, si ailleurs au Brésil le calebassier porte aussi des fruits plus petits (c'est-à-dire dont le diamètre n'excède pas cinq à dix centimètres), il n'existe aucune variété de petite calebasse au pays des Nambikuara, qui soit appropriée à la fabrication de la flûte globulaire, le diamètre de cette dernière ne pouvant excéder 10-12 $\mathrm{cm}$ pour éviter qu'elle n'épuise en peu de temps la capacité pulmonaire du musicien.

Parmi les quatre groupes Nambikuara - Halotesu, Mamaindê, Wasusu et Sararê - que nous sont familiers, les flûtes nasales sont fabriquées par les femmes, peut-être pour marquer leur différence avec les flûtes en bambou qui ne doivent jamais être aperçues et moins encore fabriquées par les femmes. Les femmes savent comment jouer de la flûte nasale, mais elles ne le font jamais. Tous les hommes, en revanche, pratiquent la flûte nasale qu'ils apprennent en bas âge, ce qui corrobore l'affirmation de Ostwald (1977: 32) selon laquelle la pratique d'un instrument de musique doit s'inculquer tôt:

«[...] nos capacités intellectuelles et sensorielles d'apprendre des mouvements compliqués tendent à diminuer après la puberté; ainsi le nombre de personnes susceptibles de se distinguer dans le domaine musical décroît».

Au même titre que la faculté d'assimiler couramment une langue étrangère comporte ses limites (deux à douze ans selon Lenneberg 1967: 159, fig. 4.4 et 4.5), la capacité d'apprendre à faire de la musique est restreinte. La méthode de Suzuki destinée à enseigner le violon aux enfants âgés entre trois et six ans est une illustration parlante de la facilité avec laquelle les enfants apprennent des actes complexes (Arakawa, communication personnelle 1981; Mills \& 
Murphy 1973). Voilà probablement la raison pour laquelle les enfants Nambikuara commencent tôt leur apprentissage musical.

Comme l'exige un usage courant de l'ethnomusicologie (Nettl 1964: 76), il nous reste à mentionner les noms que les divers groupes Nambikuara attribuent à la flûte nasale. Les Mamaindê l'appellent yelandi ou, selon l'orthographe de Price, ye ${ }^{3}$ lal $^{1} \operatorname{li}^{2}$ (s.d.: 3 infra). Aspelin a relevé un autre nom: yelathu (communication personnelle, 1971). Roquette-Pinto (1938: 350) écrit dans son glossaire du Nambikuara-Kôkôzu: hait-tea-taçu, et Boglár, dans son dictionnaire assez exhaustif du dialecte Waklitisu-Elotasu d'Utiariti, parle de maitetansu, terme repris par Oberg sous la forme de maitétansu (Boglár 1960: introduction et chapitre 10; Oberg 1953: 101), que nous avons nous-même entendu prononcer à Serra Azul en 1967.

\section{Musique et culture}

Quel est le rôle culturel de la musique chez les Nambikuara? Witherspoon (1977; voir Brady 1981: 137) a noté au sujet de la musique des Navajo que «l'expérience esthétique est à la fois intellectuelle, émotionnelle, morale, esthétique et biologique», et nous pourrions y ajouter au moins une autre fonction: culturelle. Il n'est pas facile de dégager le rôle joué par la musique en général, sans parler de la musique instrumentale en particulier, parmi les Indiens, qui se montrent très réservés en la présence d'auditeurs étrangers. Lors de nos séjours sur le terrain, nous n'avons entendu la flûte nasale que sous la forme d'une musique de divertissement, constat fait aussi par Oberg (1953: 102): «Les flûtes nasales ne semblent pas avoir de signification rituelle»; et Halmos (1979: 212) d'ajouter:

«[...] les enregistrements ont été réalisés par l'interprète seul sur la flûte nasale globulaire [en anglais: globular, mais il serait plus correct de dire: discoïde]. En ce qui concerne les fonctions de ces mélodies, nous n'en savons strictement rien».

Toutefois, l'ouvrage de Boglár nous donne une information importante sur la signification de la flûte nasale qui, selon ses informateurs, sert à attirer ou à repousser les esprits de la forêt, ou encore à appeler les hommes au travail dans les plantations. Il écrit:

«[Soudain, Masise commence à parler] 'Il y a Atasu [...] dans la forêt, dans les champs [...] je l'appelle avec la petite flûte maitetansu, mais je peux aussi le repousser [...] Masise montrera plus tard de quelle manière [...] et maintenant je veux une cigarette» (1966: 99-100);

Plus loin:

«Masise sort une flûte maitetansu de la poche de sa blouse [...] il explique comment fonctionne l'instrument et avec quels sons il attire les esprits de 
la forêt et avec lesquels il les repousse. Daniel ajoute qu'avec cette flûte plus petite, il est possible d'appeler les hommes aux travaux dans les jardins, car c'est généralement au moyen des flûtes plus grandes que l'on appelle les esprits» (1966: 124-125).

Parmi les Nambikuara-Halotesu, nous avons relevé une autre fonction de la flûte nasale. Ces Indiens entièrement «pacifiés» à l'époque de notre visite avaient noué des liens permanents avec la société environnante et commencé à vendre leurs objets en tant que curiosités. Les non-Indiens étaient généralement surpris de voir un instrument si étrange. Au moment de notre visite, ces flûtes servaient déjà d'objet d'échange. Nous pensons que l'usage de la flûte nasale comme unité de monnaie a débuté vers 1965; mais parmi les groupes Nambikuara que nous avons étudiés, seuls les Halotesu se livraient à ce commerce. Les quelques flûtes que nous avons vues chez les autres groupes n'étaient pas pyrogravées. Or, la flûte comme objet d'échange devait être joliment décorée, alors que sa qualité musicale était insignifiante, les acheteurs ne sachant pas en jouer.

\section{Les mélodies}

La transcription $N^{\circ} 1$ repose sur un enregistrement réalisé en 1966 dans le village des Mamaindê; c'est le plus long de nos enregistrements de musique de flûte nasale (durée: 2'41'). Nos connaissances linguistiques ne suffisent pas pour découvrir la fonction de cet air, mais nous croyons qu'il s'agit d'un divertissement. Nous lui avons ajouté la précision tempo giusto pour attester la virtuosité du flûtiste et la qualité technique de son instrument. Il arrive souvent que la musique instrumentale des Indiens soit jouée sur un rythme irrégulier et hésitant, et qu'elle soit interrompue par de nombreuses pauses, ce qui n'était pas le cas de la pièce en question.

La mélodie est descendante, c'est-à-dire que le premier ton est plus aigu que le dernier. Selon Kolinski (1965), la formule de niveau est 100:57, si bien que la mélodie est descendante à $43 \%$. Si nous continuons à analyser la mélodie, nous constatons que le passage marqué A-A dans la transcription est répété pas moins de treize fois, sans considérer quelques modifications minimales. Il est facile de déterminer le ton principal. Les trois tons de la mélodie apparaissent dans les rapports suivants:

$\begin{array}{lrr}\text { do } & 84 \text { fois } & 28,4 \% \\ \text { mi } b & 158 \text { fois } & 53,4 \% \\ \text { sol } b & 54 \text { fois } & 18,2 \%\end{array}$

Le mi $b$ occupe plus de la moitié de la pièce; de plus, c'est le ton final de nombreuses formules mélodiques, si bien qu'il faut le considérer comme le ton 


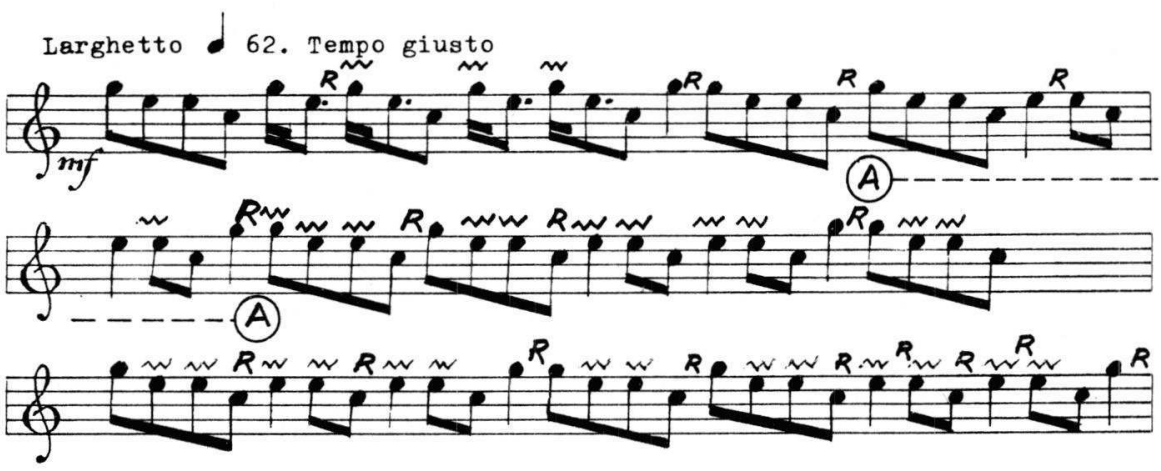

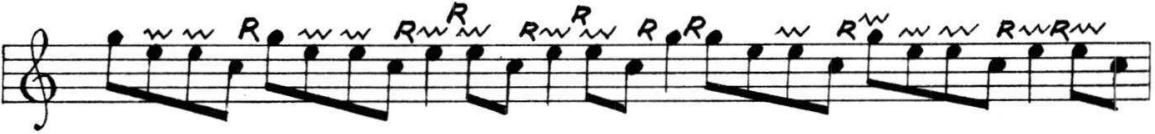

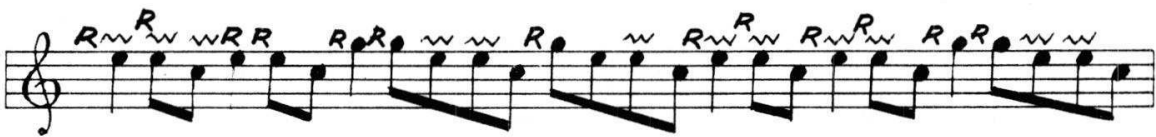
$\int e^{R}$

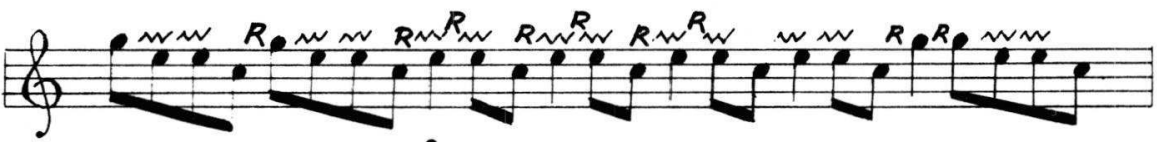
(1) REN RWR

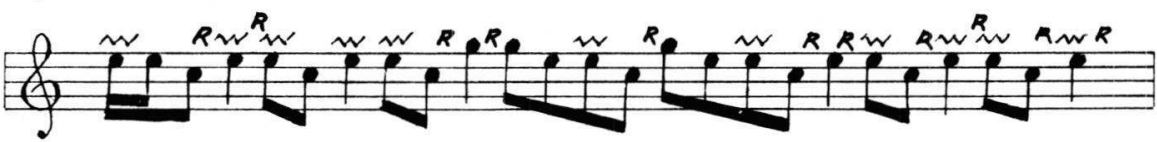

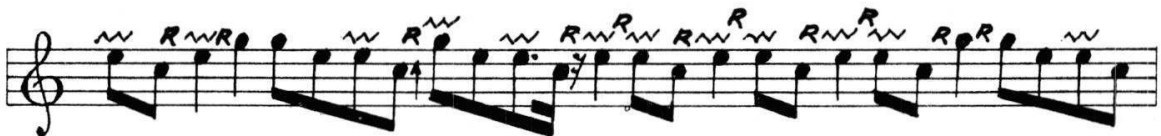

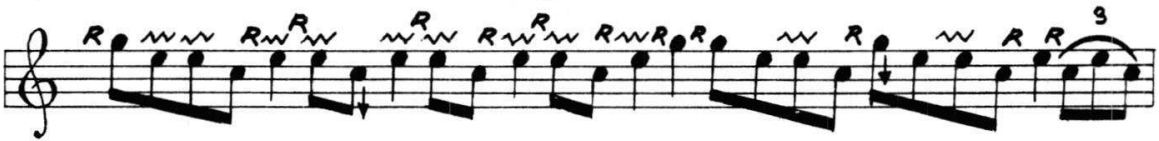

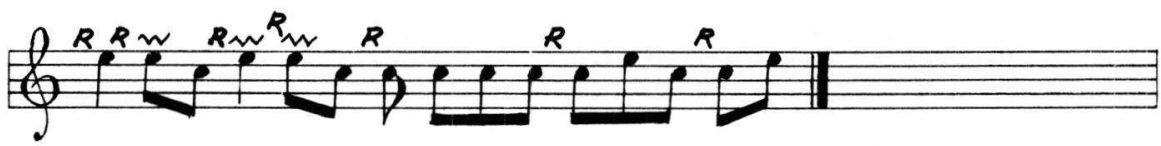


principal. Les sons très brefs et éphémères du mordente n'ont bien sûr pas été pris en considération. La lettre $\mathrm{R}$ sert à indiquer la respiration audible.
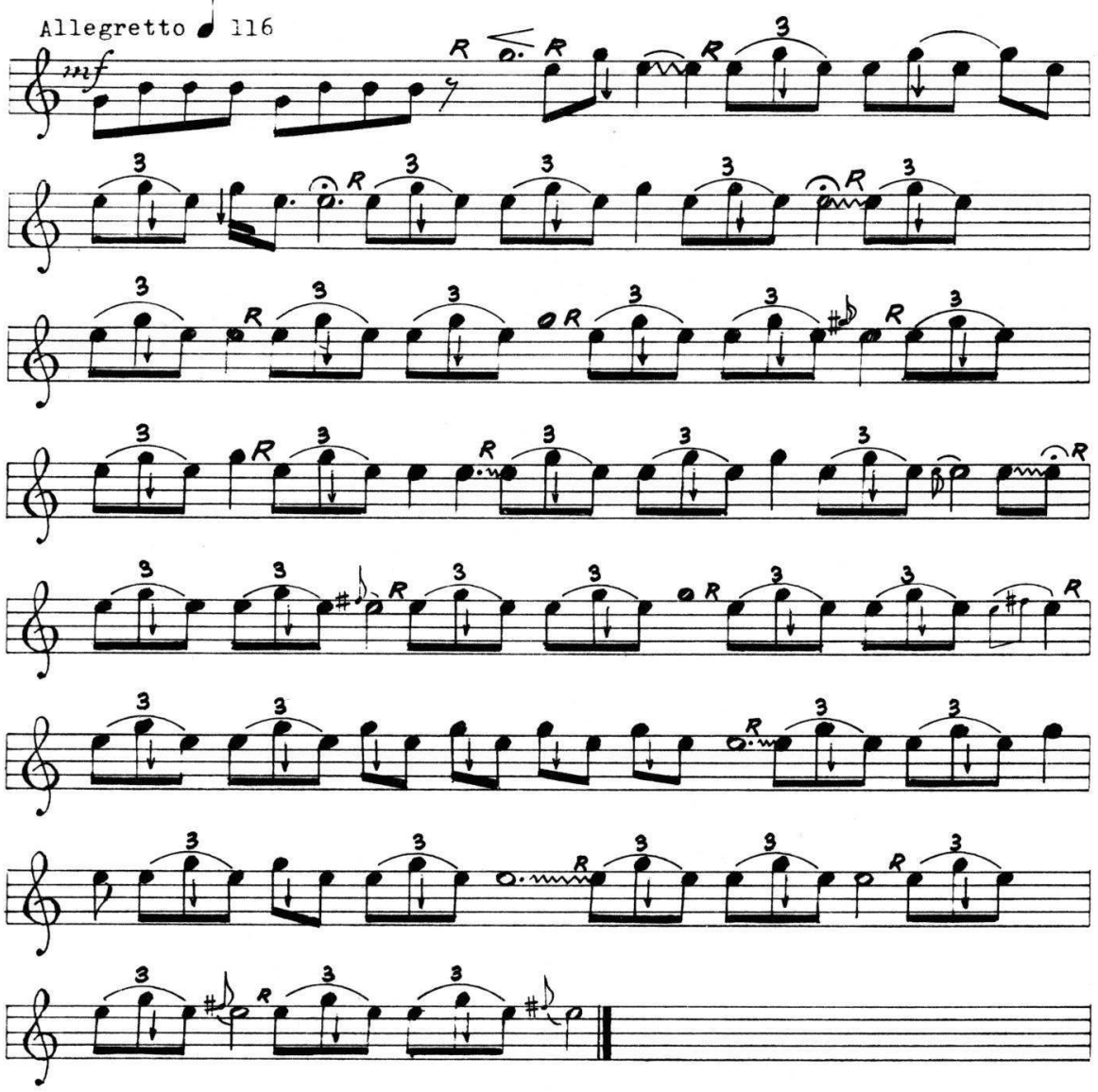

Transcription $\mathrm{N}^{\circ} 2$

La deuxième transcription provient du village nambikuara de Serra Azul et se fonde sur un enregistrement réalisé en 1967. Le musicien était Lorenzo, un virtuose de la flûte nasale. Le rythme n'est plus tempo giusto mais rubato, et la formule de niveau est 0:75, c'est-à-dire ascendant à $75 \%$. Les quatre tons de la mélodie apparaissent dans les rapports suivants (sans considérer les ornementations):

$\begin{array}{lrr}\text { sol } & 2 \text { fois } & 1,3 \% \\ \text { si } & 6 \text { fois } & 3,8 \% \\ m i & 99 \text { fois } & 62,7 \% \\ \text { sol } b & 51 \text { fois } & 32,2 \%,\end{array}$




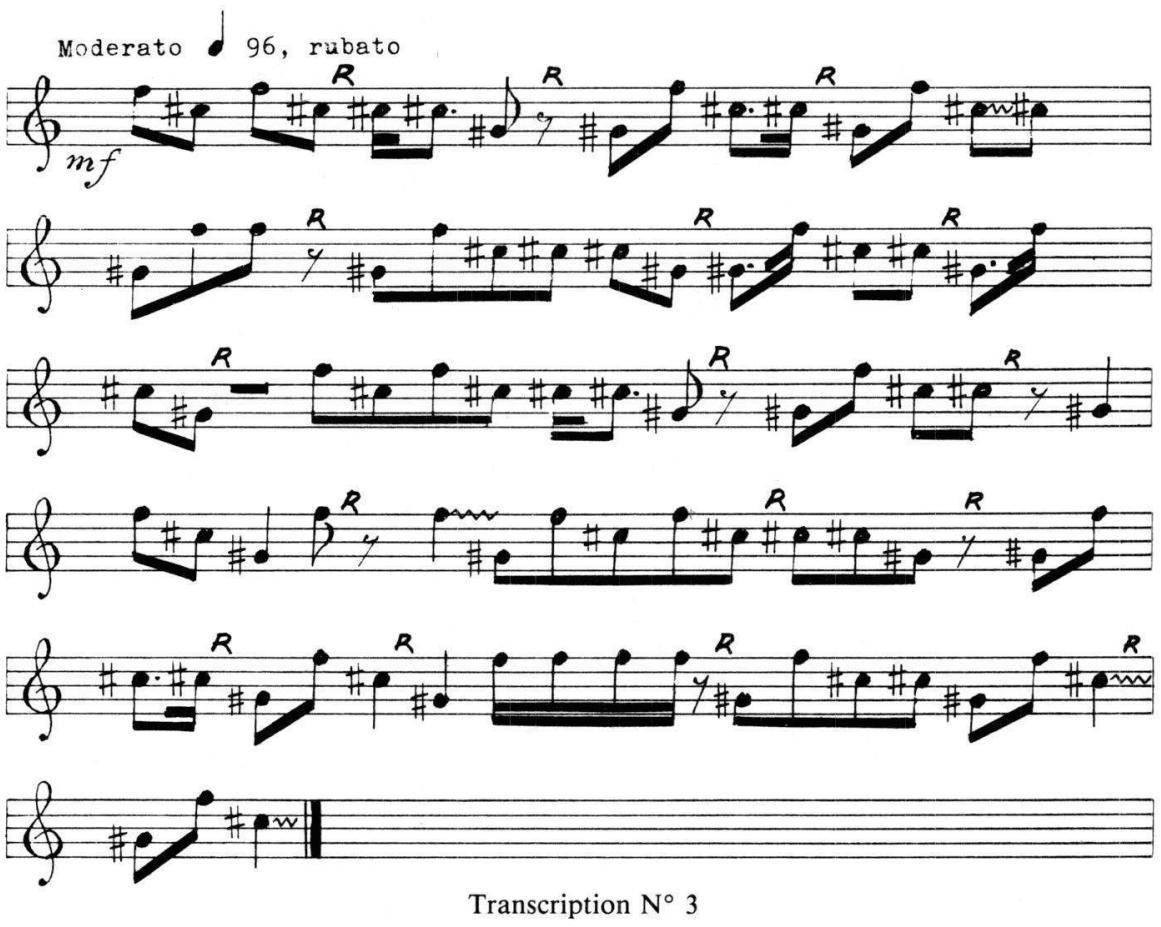

si bien que mi est le ton principal, car il est le plus fréquent et aussi le ton final. L'ambitus, soit onze demi-tons, est étendu pour une flûte nasale.

La transcription $N^{\circ} 3$ a été établie sur la base d'un enregistrement réalisé parmi les Indiens Galera ou Wasusu. Dans ce cas également, nous nous sommes aperçus combien il est difficile d'écouter de la musique indienne. Nous étions deux - ma femme et moi - à arriver au village après la traversée en camionnette d'un désert de sable redoutable s'étendant sur quarante kilomètres. Un missionnaire résidait avec sa famille à l'extérieur du village, dans une maison entourée d'une clôture; à notre question de savoir si les Indiens chantaient souvent, il répondit que ce groupe ignorait totalement la musique, car durant les huit mois qu'il se trouvait là, il n'avait entendu ni chant ni musique instrumentale.

Nous décidâmes de passer la nuit dans notre camionnette; après avoir pris notre dîner préparé sur le feu en compagnie de quelques Indiens, nous voulûmes tout de même faire une tentative pour amener les Indiens à faire de la musique que nous pourrions enregistrer. C'est ainsi que nous nous mîmes à entonner quelques chansons. Les Indiens semblaient surpris; bientôt, ils se mirent à chanter eux-mêmes. Il ne nous a pas fallu longtemps pour nous rendre compte que tout le village était assis ou debout autour de nous. A la fin, un Indien exécuta la pièce transcrite ici, dont la formule de niveau est de 0:57, 
c'est-à-dire ascendant à $57 \%$. La mélodie de cette pièce indienne n'est nullement «primitive» ni «simple», mais fort variée, et elle comporte de larges intervalles.

Nous ne sommes pas encore au clair sur la question de savoir quelle fonction attribuer aux trois mélodies de flûte nasale enregistrées. Il est peu probable qu'il s'agisse d'airs magiques. L'atasu évoqué par Boglár est un esprit maléfique susceptible d'apparaître en n'importe quel lieu et à n'importe quel moment, et le flûtiste n'oserait pas l'attirer par sa musique.

«[...] le Nambikuara se sent entouré d'esprits maléfiques. Ceux-ci étaient plus nombreux dans le passé. Aujourd'hui, grâce aux succès remportés par les 'uaníndisu' [pajé, sorcier], leur nombre a considérablement diminué [...] Les esprits maléfiques sont désignés par le terme générique d'atasu») (Pereira 1973: 3).

Il ne semble pas non plus s'agir d'airs appelant les gens au travail. C'est pourquoi nous y voyons avant tout une activité artistique ou un divertissement.

La flûte nasale des Nambikuara n'est pas utilisée pour la musique de danse. En transcrivant les airs, nous avons constaté qu'il est difficile d'y déterminer une périodicité. Comme nous, Halmos a renoncé, dans ses transcriptions encore inédites des mélodies de la flûte nasale, à indiquer le rythme. Cela ne veut cependant pas dire que cette musique soit dépourvue de pulsation; or, comme celle-ci n'est pas isopériodique, elle ne se prête pas à la danse.

Une particularité de la flûte nasale est l'impossibilité d'en intensifier le son à volonté. Notre collection comprend des instruments qui restent muets quand ils sont fortement soufflés, alors qu'il y en a d'autres qui émettent un son plus fort lorsque la hauteur de ce son augmente. Nos enregistrements ne font apparaître aucune intensification du son; or notre cerveau organise les informations reçues de manière que nous entendions une pulsation. Nous sommes d'accord avec Kolinski lorsqu'il affirme que:

«[...] le concept occidental largement répandu de mètre pourrait être qualifié le mieux par le terme d'accentuel [...] une telle approche ne s'applique pas lorsqu'une mesure commence par une pause ou une note liée. De plus, l'auditeur est parfaitement capable de reconnaître le mètre d'une pièce jouée sur l'orgue ou le clavecin sans être guidé par une accentuation» (1978: 241).

Ce paradoxe s'applique, outre aux instruments évoqués par Kolinski, à la flûte nasale. La cause doit être cherchée, selon l'auteur, dans un phénomène de la psychologie de la gestalt qui

«considère une pièce de musique comme une gestalt temporaire représentant un champ dynamique d'interaction de ses divers facteurs structurels [...] le rythme est une durée organisée, le mètre, une pulsation organisée» (1978: 241). 
Hindemith est du même avis:

«Même dans l'enchaînement de sons identiques à tout point de vue, qui se répètent avec le même rythme temporel, l'ouïe tend à retenir des groupes de sons réguliers et à donner de l'importance à certains sons plutôt qu'à d'autres, si bien que toute la série est perçue comme des vagues d'espacestemps accentués et non accentués. Ce type d'accents [accents métriques], déterminé par notre capacité sensorielle et non pas par la différence entre les sons, est indépendant de tout autre type d'accents [il est dynamique]» (1975: 53).

Nous pourrions conclure notre étude sur ces mots, mais une information due à Claude Lévi-Strauss et relative au jeu simultané de plusieurs flûtes nasales, nous invite à faire une dernière remarque. Lévi-Strauss dit: "Les flûtes nasales sont aussi jouées à l'unisson» (1948: 368). Hélas, il n'existe aucune transcription ni aucun enregistrement qui nous permette de tirer au clair cet aspect intéressant et important de l'ethnomusicologie des Nambikuara (LéviStrauss, communication personnelle 1981).

\section{Bibliographie}

\section{ALBISETTI César \& JAYME Angelo}

1962 Enciclopédia Bororo I. Campo Grande: Museu Regional Dom Bosco (publ. $\mathrm{N}^{\circ}$ 1).

AYTAI Desidério

1967 "As flautas rituais dos Nambikuara». Revista de Antropologia (São Paulo) 15/16: 69-75.

1981 «A música instrumental xavante». Latin American Music Review (Austin) 2(1): 103-129.

BOGLÀR I ajos

1960 «Nambikuara vocabulary». Acta Ethnographica Academiae Scientiarum Hungaricae (Budapest) IX(1-2): 89-117.

1966 Trópusi indiánok között [Chez les Indiens des Tropiques]. Budapest: Gondolat.

BOGLÀR Lajos \& HALMOS István

1962 «La flûte nasale chez les Indiens Nambicuara». Acta Ethnographica Academiae Scientiarum Hungaricae (Budapest) XI(3-4): 437-46.

BRADY Margaret $\mathrm{K}$.

1981 «Book review of Witherspoon's 'Language and art in the Navajo universe'». Ethnomusicology XXV(1): 136 .

HALMOS István

1979 "The music of the Nambicuara Indians». Acta Ethnographica Academiae Scientiarum Hungaricae (Budapest) 28(1-4): 205-350. 
HINDEMITH Paul

1975 Treinamento elementar para músicos. Traduit par M. Camargo Guarnieri. São Paulo: Ricordi.

IZIKOWITZ Karl Gustav

1935 Musical and other sound instruments of the South American Indians. Goteborg: Elanders Boktryskeri Aktiebolag.

KOLINSKI Mieczyslaw

1965 «The general direction of melodic movement». Ethnomusicology IX(3): 240-265.

1978 «The structure of music: diversification versus constraint». Ethnomusicology XXII(2): 229-244.

KOZÀK Vladimir, BAXTER David, WILLIAMSON Laila \& CARNEIRO Robert L.

1979 The Héta Indians: fish in a dry pond. New York: American Museum of Natural History (Anthropological Papers 55(6)).

LAMING-EMPERAIRE Annette, MENEZES Maria José \& ANDREATTA Margarida D.

1978 O trabalho de pedra entre os Xetá. São Paulo: Univ. de São Paulo (Coleção Museu Paulista, Série Ensaios $N^{\circ} 2$ ).

LENNEBERG Eric H., CHOMSKY Noam \& MARX Otto

1957 Biological foundations of language. New York: John Wily \& Sons, Inc.

LÉVI-STRAUSS Claude

1948 «The Nambicuara». In: Handbook of South American Indians III. Washington, D.C.: Bureau of American Ethnology.

MILLS Elisabeth \& MURPHY Therese Cecile Sr

1973 The Suzuki concept. Berkeley: Diablo Press.

NETTL Bruno

1964 Theory and method in etnnomusicology. New York: The Free Press.

OBERG Kalervo

1953 Indian tribes of the Northern Mato Grosso, Brazil. Washington D.C.: Smithsonian Institution.

OSTWALD Peter

1977 «Psicanálise do som». Correio da UNESCO 4(1): 5-13.

PEREIRA Pe. Adalberto Holanda, S.J.

1973 Os espíritos maus dos Nambikuára. São Leopoldo: Instituto Anchietano de Pesquisas (Antropologia $\mathrm{N}^{\circ} 25$ ).

PUTTKAMER W. Jesco von

1979 «Man in the Amazon: Stone Age present meets Stone Age past». National Geographic 155(1): 60-82.

ROQUETTE-PINTO E.

1938 Rondônia. São Paulo: Co. Editora Nacional (Brasiliana, 5[a], vol. 39).

SCHMIDT Max

1912 «Reisen in Matto Grosso». Zeitschrift für Ethnologie XLIV: 130-174.

WEINER J.S. \& LOURIE J.A.

1969 Human biology. Oxford/Edinburgh: Blackwell Scientific Publications (International Biological Programme Handbook ${ }^{\circ} 9$ ).

WITHERSPOON Gary

1977 Language and art in the Navajo universe. Ann Arbor: University of Michigan Press. 